\title{
The sensitivity of warm period precipitation forecasts to various modifications of the Kain-Fritsch Convective Parameterization scheme
}

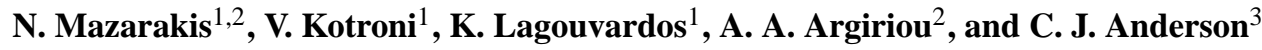 \\ ${ }^{1}$ National Observatory of Athens, Athens, Greece \\ ${ }^{2}$ University of Patras, Department of Physics, Patras, Greece \\ ${ }^{3}$ Iowa State University, Climate Science Initiative, Ames, Iowa, USA
}

Received: 5 May 2010 - Revised: 30 September 2010 - Accepted: 18 February 2011 - Published: 12 May 2011

\begin{abstract}
The sensitivity of quantitative precipitation forecasts to various modifications of the Kain-Fritsch (KF) convective parameterization scheme (CPS) is examined for twenty selected cases characterized by intense convective activity and widespread precipitation over Greece, during the warm period of 2005-2007. The study is conducted using the MM5 model with a two nested domains strategy, with horizontal grid increments of 24 and $8 \mathrm{~km}$, respectively. Five modifications to the KF CPS, each designed to test the sensitivity of the model to the convective scheme formulation, are discussed. The modifications include: (i) the maximization of the convective scheme precipitation efficiency, (ii) the change of the convective time step, (iii) the forcing of the convective scheme to produce more/less cloud material, (iv) changes to the trigger function and (v) the alteration of the vertical profile of updraft mass flux detrainment.

The simulated precipitation from the $8-\mathrm{km}$ grid is verified against raingauge measurements. Although skill scores vary widely among the cases and the precipitation thresholds, model results using the modifications of the convective scheme show improvements in 6-h precipitation totals compared to simulations generated using the unmodified convective scheme. In general, forcing the model to produce less cloud material improves the precipitation forecast for the moderate and high precipitation amounts, while the same modification and the change of the convective time step to $1 \mathrm{~min}$ has the same result for the high precipitation thresholds. The increase of convective time step to $15 \mathrm{~min}$, the maximization of precipitation efficiency and the changes to the trigger function give similar results for medium and high precipitation. On the other hand, the forecast for the light
\end{abstract}

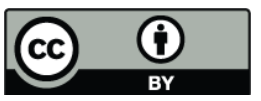

Correspondence to: N. Mazarakis (nickmaz@meteo.noa.gr) precipitation is improved by forcing the model to produce more cloud material as well as by the alteration of the vertical profile of updraft mass flux detrainment.

\section{Introduction}

Greece is a topographically diverse country; thus, there are many variations in the weather conditions especially during the warm period of the year. Typically, the climate during summer and early autumn is characterized by high temperatures and limited amounts of rainfall. However, during this period, many days are characterized by intense convection and important lightning activity especially over the northern part of the country (Mazarakis et al., 2008). Because of the small scale of these phenomena, numerical models frequently fail to provide acceptable quantitative precipitation forecasts. Convection cannot be explicitly resolved at the resolution used by operational mesoscale models when the resolution is greater than $4 \mathrm{~km}$, and thus it is parameterized using one of the various convective parameterization schemes (CPS). Many studies have shown that among the various CPS used in the numerical weather prediction (NWP), the KainFritsch (KF) scheme (Kain and Fritsch, 1990, 1992) performs well in forecasts of convective systems (Kuo et al., 1996; Wang and Seaman, 1997; Kotroni and Lagouvardos, 2001, 2004; Ferrier, 2004; Mazarakis et al., 2009).

The implementation of the KF scheme is characterized by a tendency in some cases to overpredict precipitation amounts (Spencer and Stensrud, 1998; Gallus and Segal, 2001; Correia et al., 2008). Other problems are the erroneous placement of the convective activity and the failure of the representation of propagating convection (Davis et al., 2003). In particular, the quantitative precipitation forecast (QPF) above the Greek peninsula mainly suffers from

Published by Copernicus Publications on behalf of the European Geosciences Union. 
the two aforementioned problems (Kotroni and Lagouvardos, 2004; Mazarakis et al., 2009).

Numerous studies have been devoted to the improving the performance of the KF CPS. Some of them focus on the change of several simple tunable parameters of the scheme while others are more sophisticated, as they consist of major changes to the core of the scheme. The main modification of the initial scheme (Kain and Fritsch, 1990) included major changes to the updraft and downdraft formulation, allowing shallow (nonprecipitating) convective clouds and also changes to the closure assumption (Kain, 2004). Until then, various simpler modifications had been implemented. For example, Spencer and Stensrud (1998) have tried to improve the model skill of QPF by applying three different modifications. These modifications included the maximization of the precipitation efficiency, the elimination of the convective downdrafts below the cloud base and the delay of the downdrafts relative to the initialization of the updrafts. Model simulations using each one of these modifications showed an improvement in the estimation of total rainfall fields versus the simulations that use the unmodified convective scheme, since the rainfall maxima provided by the model were often near the observed maxima, especially when the second modification was been used. In another study, Gallus and Segal (2001) used the KF scheme with the ETA model for twenty warm mesoscale convective system (MCS) cases and made various initialization adjustments. For their study they used two different convective time steps, $10 \mathrm{~min}$ and $20 \mathrm{~min}$, and found that doubling the convective time step did not have any significant impact on the precipitation forecasts.

Many authors focused on the modification of the physical characteristics of the updrafts and downdrafts in the KF scheme. For example, Anderson et al. (2002) tried to improve the QPF skill in two cases characterized by elevated convection by implementing two different modifications; the downdraft mass flux was related to the mean relative humidity within the downdraft layer and the cloud radius varied between 100 and $3000 \mathrm{~m}$. Ferrier (2004) managed to alleviate the problem of high QPF bias, testing various modifications of the KF CPS and found some improvements in QPF. Namely, in his modifications (i) all of the rain and snow calculated in the updrafts are detrained onto the grid, in which all subsequent cloud and precipitation processes are calculated by the grid-scale microphysics, (ii) fields of hydrometeors calculated by the model are used as input into the scheme and are modified by the convective processes, (iii) hydrometeors, except for rain, are converted back to cloud water, (iv) convective downdrafts are turned off and (v) the updraft radius is a function of cloud-base vertical motion. Recently, Correia et al. (2008) has used a modified version of KF CPS proposed by Anderson et al. (2007) in order to examine the sensitivity to the convection time step, the effects of hydrometeors feedback and the importance of the vertical heating-cooling profile in cases of propagating convection during the occurrence of MCSs. This modification differs from the original $\mathrm{KF}$ in that updraft detrainment of heat, moisture, and condensate begins above the level of minimum saturated equivalent potential temperature, whereas the KF CPS begins detraining above the level of equilibrium temperature. Using the aforementioned modification, they carried out numerous sensitivity experiments (i) altering the CPS microphysics to produce less convective precipitation, (ii) modifying the downdraft temperature effects in the lowest levels, (iii) using two alternative convective time steps ( $1 \mathrm{~min}$ and $4 \mathrm{~min}$ ), and (iv) testing three idealized profiles to determine the sensitivity to the magnitude and shape of the heating. As a result of these modifications, stronger gravity waves were produced that lead to the production of spurious convection. The results suggested that two processes are of fundamental importance for propagation: depth of low-level cooling and the location of the heating maximum.

One of the most important parameters in convective schemes is the "trigger function" (Kain and Fritch, 1992) because its formulation and the criteria that should be satisfied play a significant role in the scheme activation. Modification of the "trigger function" can have a positive impact on the QPF skill. For example, a modified version of the KF CPS has been used by Ridout et al. (2005) in which a cloud base quasi-balance constraint on convective cloud-base mass flux is implemented. The modified scheme includes changes to the updraft source-layer selection and to the convective trigger perturbations, while a great part of this is based on the Emanuel convective scheme (Emanuel, 1991; Emanuel and Zivkovic-Rothman, 1999). More recently, Truong et al. (2009), using a modified version of Kain-Fritch CPS, succeeded at an important improvement in simulated rainfall compared to the original scheme for a case of serious flooding in Central Vietnam. A new diagnostic equation to compute updraft velocity, closure assumption and trigger function was used in their modification where they take the vertical gradient of the Exner function perturbation into account, with an on-off coefficient to account for the role of the advective terms. Thanks to the analytical computation of the ratio between the vertical gradient and buoyant forces for the updrafts, they created different expression than the original for the trigger function, updraft velocity and CAPE.

In the present study, a mesoscale model skill to simulate 20 warm season events, characterized by intense convective activity and widespread precipitation, is investigated. These events affected the Greek Peninsula during the warm season period of 2005-2007. Since during the warm period of the year most part of the precipitation is convective, it was considered important to focus on the performance of CPS used by the model in order to improve the QPF. More specifically, the model sensitivity to various modifications of the KF CPS scheme has been explored. These modifications include (i) the maximization of the convective scheme precipitation efficiency, (ii) the change of the convective time step, (iii) the forcing of the convective scheme to produce more/less cloud material, (iv) changes to the trigger function 


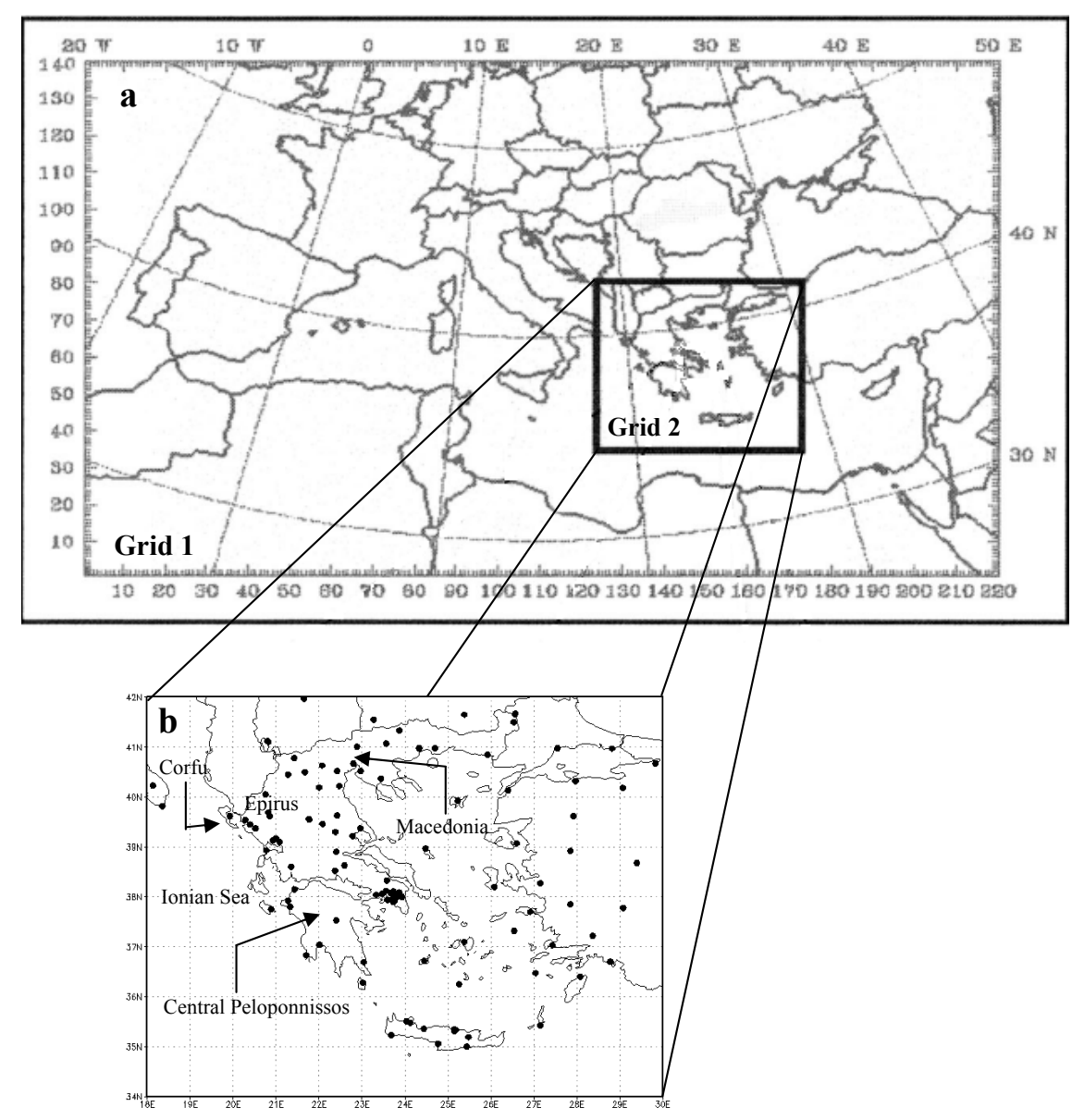

Fig. 1. (a) MM5 nested model domains. (b) Location of the 113 rain gauges (marked by black circles) used in the verification procedure.

and (v) the alteration of the vertical profile of updraft mass flux detrainment. The results of the performed simulations have been compared with the available raingauge observations with the aim to statistically evaluate the model performance for each modification.

The paper is arranged in the following manner: in Sect. 2 the three-dimensional numerical model is described in brief, while the selected modifications to the KF CPS scheme are discussed in Sect. 3. The available data and the verification methodology are presented in Sect. 4. The results of QPF verification are presented in Sect. 5 while an example of a case study is given in Sect. 6. Section 7 is devoted to the summary and discussion of this work.

\section{Model setup}

The numerical model used for this study is the MM5 (version 3), a nonhydrostatic primitive equation model using terrain-following coordinates (Dudhia, 1993). Several physical parameterization schemes are available in the model for the boundary layer turbulence, the radiative transfer, the microphysics, and the cumulus convection. The MM5 model is running operationally at the National Observatory of Athens since 2000 (Kotroni and Lagouvardos, 2004). Concerning the choice of the microphysical and boundary layer schemes, the current operational chain uses the scheme proposed by Schultz (1995) and Hong and Pan (1996), respectively. Indeed, in the study by Akylas et al. (2007), the authors have shown that the PBL scheme proposed by Hong and Pan (1996) produces the most consistent results over Greece. For radiation parameterisation, the standard scheme of MM5 that accounts for longwave and shortwave interactions with explicit cloud and clear-air was used. Finally, a five-layer soil model scheme was selected.

Two one-way nested grids are defined and used in this study (Fig. 1a). Grid 1 has a 24-km horizontal grid increment, covering the major part of Europe, the Mediterranean, and the northern African coast. Grid 2 has a 8-km horizontal grid increment, covering the Greek territory and all the Greek islands. The horizontal extension of the defined operational grids is shown in Fig. 1a. In the vertical direction, 23 unevenly spaced full sigma levels are selected with the spacing of levels reduced near the ground surface to better simulate the evolution of the planetary boundary layer. 
MM5 is initialized at 00:00 UTC for every nested grid and the simulation lasts $24 \mathrm{~h}$. The 00:00 UTC Global Forecast System (GFS), provided by the National Centers for Environmental Prediction (NCEP), gridded analysis fields and 6h-interval forecasts are used to initialize the model and nudge the boundaries of Grid 1 during the simulation period. No preforecast spinup period or assimilation of additional observations is used in the operational MM5 chain.

In addition to the aforementioned mode chain (CTRL hereafter), where the modified from Kain KF scheme version is used (Kain, 2004), eight additional experiments have been made for each case study, keeping all other model settings the same, except the configuration of KF CPS. As this study focusses on the sensitivity of warm season precipitation forecasting and on the choice of the modification of KF CPS, twenty days of the warm period of 2005-2007 have been selected and simulated using the original and the KF scheme modifications. Thus, one hundred eighty simulations have been performed in total.

\section{Modifications to the Kain-Fritch CPS}

The KF CPS receives the model column values of temperature, specific humidity, vertical and horizontal wind speed, and pressure from the host model. In addition, the scheme returns tendencies applied to the gridpoint values of temperature, specific humidity, cloud liquid water, and cloud ice. The core of the scheme is a Lagrangian model of a onedimensional entraining-detraining steady-state plume with downdraft to compute convective tendencies such that CAPE of the model column is reduced by $90 \%$. A complete description of the KF CPS is found in Kain and Fritsch (1993), Bechtold et al. (2001) and Kain (2004). The modifications that have been used in the present study are described in the following paragraphs.

\subsection{Maximizing Precipitation Efficiency (PEF09)}

The first modification to the KF CPS is to maximize the precipitation efficiency (PE), which is simply the ratio of the water mass reaching the ground as precipitation to the water vapor mass entering the cloud. In the original scheme, the PE is expressed as a function of (i) the vertical shear of the horizontal wind evaluated over the cloud depth and (ii) the height of cloud base (Fritch and Chappell, 1980; Kain and Fritch, 1990) and is restricted to values $0.1 \leq \mathrm{PE} \leq 0.9$. To maximize rainfall from the convective scheme, the PE is taken equal to 0.9 whatever the wind conditions and cloud base height. This modification forces $90 \%$ of the water vapor influx at low levels to fall as precipitation, thereby minimizing the amount of liquid water available to drive evaporatively cooled convective downdrafts and maximizing the convective rainfall each time the convective scheme is activated (Spencer and Stensrud, 1998).

\subsection{Alteration of the convective time step (1MIN, 15MIN)}

The second and third modifications are relatively simple and consist in the alteration of the period at which the model calls the convective parameterization scheme, namely the convective time step. In the original scheme, the convective initiation is checked every $5 \mathrm{~min}$. In this study two different alterations have been considered. In the first, the convective time step is taken equal to one minute and in the second, equal to $15 \mathrm{~min}$. Yang and Arritt (2001) found in regional climate simulations that adjustments in this parameter could have a pronounced impact on precipitation forecast. In their study, the CPS proposed by Grell (1993) and Grell et al. (1994) was used. On the contrary, the doubling of that time step had limited impact in the study of Gallus and Segal (2001), where the KF and Betts-Miller-Janjic schemes were used.

\subsection{Reduce and enhance KF precipitation (RATE01, RATE0001)}

In the original scheme the calculation of precipitation is based on the following equation:

$\delta Q_{\mathrm{v}}=Q_{\mathrm{v}}\left[1-\exp \left(\operatorname{RATE} \frac{\mathrm{dz}}{w}\right)\right]$,

where $\delta Q_{\mathrm{v}}$ is the condensate removed from the updraft, $Q_{\mathrm{v}}$ is the condensate, $\mathrm{dz}$ the depth of the model layer, $w$ the mean vertical velocity and RATE is a constant equal to the rate at which cloud droplets are converted to raindrops (Ogura and Cho, 1973). In the original scheme, RATE is equal to 0.01 . By changing the RATE to -0.1 , the convective scheme is forced to produce more rainfall and less cloud material. On the contrary, by changing the RATE to -0.001 , the convective parameterization is forced to produce more cloud material. This is useful in cases characterized by long-lived and organized convective systems.

\section{Changes to the trigger function (EASYTRIG1, EASYTRIG2)}

In the original KF CPS the convective parameterization is triggered when the trigger function $T_{\mathrm{LCL}}+\delta T_{\mathrm{VV}}-T_{\mathrm{ENV}}>$ 0 is satisfied, where $T_{\mathrm{LCL}}$ is the temperature at the Lifting Condensation Level, $T_{\mathrm{ENV}}$ the environmental temperature and $\delta T_{\mathrm{VV}}$ the temperature perturbation. This temperature perturbation is used to eliminate most parcels from further consideration by testing weather the temperature of the mixed parcels at their LCL plus $\delta T_{\mathrm{VV}}$ is greater than the environmental temperature $\left(T_{\mathrm{ENV}}\right)$ at the mixed parcel LCL height $\left(Z_{\mathrm{LCL}}\right)$. Thus, this temperature is defined as $\delta T_{\mathrm{VV}}=k\left[w_{\mathrm{g}}-c(z)\right]^{1 / 3}$ where $k$ is a constant and $c(z)=0.02 \mathrm{~m} \mathrm{~s}^{-1}$ if the mixed parcel LCL height is higher than $2000 \mathrm{~m}$ a.g.l. and $c(z)=0.02\left(Z_{\mathrm{LCL}} / 2000\right)$ if the mixed parcel LCL is lower than $2000 \mathrm{~m}$ a.g.l. In general, the implementation of the KF CPS during the warm period of the year 
over Greece suffers from small values of Frequency Bias (see Sect. 4.2 for the definition of Frequency Bias), especially for moderate and large amounts of rain (Mazarakis et al., 2009). To alleviate this effect, the coefficient (a) 0.5 (EASYTRIG1) and (b) 1.0 (EASYTRIG2) has been added to the left-hand side of the trigger function, forcing the scheme to be triggered more easily. Thus the new form of the trigger function is $T_{\mathrm{LCL}}+\delta T_{\mathrm{VV}}-T_{\mathrm{ENV}}+\Delta T>0$, where $\Delta T$ is equal to 0.5 or 1.0 .

\subsection{An alternative mass flux profile (CJAmod)}

The last modification is the more complex in comparison with those already mentioned modifications. The standard version of KF2 imposes a linear in pressure decrease of updraft mass flux from its value at the level of equilibrium temperature (LET) to zero at cloud top. In the last modification two main changes have been made. First, the bottom of the outflow layer has been changed from the LET to the level of minimum gridpoint $\theta_{\mathrm{e}}\left(\min \theta_{\mathrm{e}}\right)$ or the melting level (ML), whichever is at a lower altitude, and this level is required to be above the LCL. Second, the updraft mass flux is assumed to decrease linearly with the natural logarithm of pressure rather than with pressure. Thus, these two modifications have two main impacts. First, warming is reduced near the tropopause, because compensating subsidence occurs over a deeper layer and second, liquid and ice mixing ratios are increased in the middle portion of the troposphere. Thus, there is much more cloud water for the convective parameterization to feedback to the host model. This modification was proposed and implemented to the WRF model by Anderson et al. (2007).

\section{Data sets and verification methodology}

\subsection{Data sets}

The model verification is perfomed for twenty days characterized by intense convective activity and widespread precipitation that occurred from 15 May to 30 September of the years from 2005 to 2007. The choice of this period of the year is based on the fact that it is characterized in general by a weak synoptic-scale forcing, there is little influence from midlatitude systems and the convective activity is important over central and northern Greece (Flocas, 1993; Trigo et al., 2002; Kostopoulou and Jones, 2007; Mazarakis et al., 2008). For the statistical evaluation, the precipitation observations from 41 rain gauges operated by the Hellenic National Meteorological Service (HNMS), 26 rain gauges operated by the National Observatory of Athens, 13 rain gauges operated by the Ministry of Rural Development, 10 rain gauges operated by the National Technical University of Athens and 23 rain gauges provided by the ECMWF MARS data base are used. So the total maximum number of stations is 113 but the number of stations used for each case was case dependent.

\subsection{Statistics}

For each case the 6-h-accumulated precipitation (from $t+12$ up to $t+18$ ) were verified against 70-90 rain gauges available on average per case. This time period was selected because summer thunderstorm activity usually occurs near the time of the maximum surface heating. Indeed, inspection of the cases showed that in all selected cases, the maximum of precipitation was observed during the period 12:00 18:00 UTC while outside this time interval the observed rainfall was quite sparse. Model-simulated precipitation for the $8-\mathrm{km}$ grid was used. Following the WMO report about the verification of precipitation forecasts (WMO, 2008), a contingency table for the observed and forecasted values was built, as shown in the following:

\begin{tabular}{cc|cc}
$2 \times 2$ Contingency Table & \multicolumn{2}{|l}{ Event Observed } \\
\hline \multirow{3}{*}{ Event Forecasted } & Yes & A & No \\
& No & C & B
\end{tabular}

where $\mathrm{A}$ is the number of stations for which the model forecasted precipitation and the observed precipitation equaled or exceeded a threshold (hits), B is the number of stations for which only the model forecasted precipitation equaled or exceeded a threshold (false alarm), $\mathrm{C}$ is the number of the stations for which only the observed precipitation equaled or exceeded a threshold (misses) and D is the number of the stations for which neither the model forecasted precipitation nor the observed precipitation equaled or exceeded a threshold (correct negatives). The following measures are calculated:

- Frequency Bias, $B=\frac{A+B}{A+C}$

that gives the ratio of the forecast rain frequency to the observed rain frequency.

- Probability Of Detection, $\mathrm{POD}=\frac{\mathrm{A}}{\mathrm{A}+\mathrm{C}}$ which measures the fraction of observed events that were correctly forecasted.

- False Alarm Ratio, FAR $=\frac{B}{A+B}$

which gives the fraction of forecast events that were observed to be non-events.

- Critical Success Index, CSI $=\frac{\mathrm{A}}{\mathrm{A}+\mathrm{B}+\mathrm{C}}$

also known as the threat score which gives the fraction of all events forecasted and/or observed that were correctly diagnosed.

- Heidke Skill Score, $H S S=\frac{A+D-E}{A+B+C+D-E}$

where $\mathrm{E}=\frac{(\mathrm{A}+\mathrm{B})(\mathrm{A}+\mathrm{C})+(\mathrm{B}+\mathrm{D})(\mathrm{C}+\mathrm{D})}{\mathrm{A}+\mathrm{B}+\mathrm{C}+\mathrm{D}}$ which measures the increase in proportion correct for the forecast system, relative to that of random chance. 
In the framework of this study, the aforementioned statistical scores were calculated for five distinct thresholds of rainfall amount: $0.1,1,2.5,5$ and $10 \mathrm{~mm}$. In addition, the following quantitative measures have been calculated:

$$
\text { - mean error, } \mathrm{ME}=\frac{1}{N} \sum_{i=1}^{N}\left(F_{i}-O_{i}\right)
$$

where $F_{i}$ indicates the forecast value and $O_{i}$ indicates the observed value and $N$ is the number of observing stations. The mean error measures the average difference between the forecast and the observed values, and

$$
\text { - mean absolute error, MAE }=\frac{1}{N} \sum_{i=1}^{N}\left|F_{i}-O_{i}\right|
$$

which measures the average magnitude of the error.

ME and MAE were calculated for five ranges: 0.1-2.5, 2.5$5,5-10,10-20$, and $>20 \mathrm{~mm}$. All of the aforementioned skill scores were averaged for all cases and the results are discussed in the following section.

\section{Verification results}

Table 1a shows the Frequency Bias (B) scores for the forecasts implementing the original KF scheme and the eight different modifications as a function of the selected precipitation threshold. In general, irrespective of the modification, the model presents a tendency to overpredict the light precipitation $(\leq 1.0 \mathrm{~mm})$ and to underpredict the medium and heavy precipitation $(>5.0 \mathrm{~mm})$. However, when the model is forced to produce more precipitation and less cloud material, i.e. setting the RATE equal to -0.1 , the B presents a clear improvement, especially for the threshold of $5.0 \mathrm{~mm}$ with a score of 0.95 .

The positive effect of this modification is obvious, not only for the B but also for the CSI score (Table 1b), for medium $(>2.5 \mathrm{~mm})$ and heavy $(>5.0 \mathrm{~mm})$ precipitation. The implementation of the RATE01 modification gives 0.54 and 0.41 for the threshold of $>2.5 \mathrm{~mm}$ and $>5.0 \mathrm{~mm}$ respectively compared to - and - respectively for the CTRL. The increase of cloud material, by setting the parameter RATE equal to 0.001 , seems to have a positive effect for rain/no-rain threshold $(<0.1 \mathrm{~mm})$, resulting in the best score (0.69) among all modifications and the CTRL run. On the other hand, this modification gives poor results for the other thresholds, especially for medium and heavy precipitation. Although for the $1.0 \mathrm{~mm}$ threshold, the differences between the various modifications are indiscernible, the increase of the convective time step to $15 \mathrm{~min}$ gives slightly better results $(0.60)$, from the others. On the contrary, for heavy precipitation $(>10 \mathrm{~mm})$, the decrease of this time step to $1 \mathrm{~min}$ has a positive effect to CSI (0.25). In comparison with the aforementioned CSI skill scores, HSS follows the same pattern (Table 1c). Thus,
Table 1. (a) Frequency Bias, (b) CSI, (c) HSS, (d) POD, (e) FAR, (f) MAE, and (g) ME for the 6-h precipitation forecasts provided by the MM5 model by using the original KF scheme and the eight different modifications, averaged for the 20 selected cases. The best statistical score for every threshold is given in bold.

\begin{tabular}{lccccc}
\hline (a) Bias & 0.1 & 1 & 2.5 & 5 & 10 \\
\hline KF-CTRL & 1.09 & 1.14 & 0.97 & 0.80 & 0.50 \\
PEF09 & 1.09 & 1.13 & $\mathbf{0 . 9 8}$ & 0.79 & 0.59 \\
RATE01 & 1.05 & 1.12 & 1.08 & $\mathbf{0 . 9 5}$ & $\mathbf{0 . 7 0}$ \\
RATE0001 & 1.14 & 0.92 & 0.63 & 0.43 & 0.42 \\
EASYTRIG1 & 1.21 & 1.21 & 1.04 & 0.85 & 0.61 \\
EASYTRIG2 & 1.22 & 1.22 & 1.05 & 0.86 & 0.55 \\
1MIN & 1.18 & 1.17 & 0.99 & 0.79 & 0.47 \\
15MIN & $\mathbf{1 . 0 5}$ & $\mathbf{1 . 0 7}$ & 1.03 & 0.74 & 0.41 \\
CJAmod & 1.36 & 1.12 & 0.78 & 0.48 & 0.39 \\
\hline (b) CSI & 0.1 & 1 & 2.5 & 5 & 10 \\
\hline KF-CTRL & 0.61 & 0.57 & 0.52 & 0.39 & 0.21 \\
PEF09 & 0.63 & 0.59 & 0.49 & 0.35 & 0.24 \\
RATE01 & 0.61 & 0.59 & $\mathbf{0 . 5 4}$ & $\mathbf{0 . 4 1}$ & 0.22 \\
RATE0001 & $\mathbf{0 . 6 9}$ & 0.54 & 0.34 & 0.22 & 0.17 \\
EASYTRIG1 & 0.63 & 0.58 & 0.51 & 0.36 & 0.20 \\
EASYTRIG2 & 0.62 & 0.57 & 0.50 & 0.36 & 0.20 \\
1MIN & 0.63 & 0.59 & 0.51 & 0.38 & $\mathbf{0 . 2 5}$ \\
15MIN & 0.64 & $\mathbf{0 . 6 0}$ & 0.51 & 0.39 & 0.24 \\
CJAmod & 0.61 & 0.59 & 0.44 & 0.28 & 0.15 \\
\hline
\end{tabular}

RATE0001 gives the best results for the rain/no-rain threshold (0.71) while RATE01 is clearly better than the other modification for the thresholds of $>2.5 \mathrm{~mm}$ and $>5.0 \mathrm{~mm}$ with scores 0.62 and 0.52 , respectively. Similar to CSI, for the heavy precipitation $(>10 \mathrm{~mm})$, the reduction of the time step from $5 \mathrm{~min}$ to $1 \mathrm{~min}$ noticeably improves the forecast.

As far as the probability of detection (POD) is concerned (Table 1d), CJAmod gives the best results for rain/no-rain threshold while RATE01 gives the best results for medium and heavy precipitation. Especially for the $>2.5 \mathrm{~mm}$ and $>5.0 \mathrm{~mm}$, there is a clear predominance of the RATE01 with POD equal to 0.72 and 0.57 , respectively. The results for the threshold of $10 \mathrm{~mm}$ are identical between RATE01 and PEF09, while the modification EASYTRIG1 gives slightly better results for the threshold of $1.0 \mathrm{~mm}$. Concerning the false alarm ratio (FAR) shown in Table 1e, RATE0001 shows better skill than the other modifications for rain/norain threshold as FAR is closer to 0 (perfect score). The alteration of the convective time step seems to have a positive effect for medium and heavy precipitation because $1 \mathrm{MIN}$ and $15 \mathrm{MIN}$ are closer to 0 .

The aforementioned scores only give a measure of the model accuracy based on the frequency of precipitation occurrence at or above a threshold and do not account for the magnitude of precipitation errors. Investigation of the quantitative bias of forecast precipitation is performed through 
Table 1. Continued.

\begin{tabular}{|c|c|c|c|c|c|}
\hline (c) HSS & 0.1 & 1 & 2.5 & 5 & 10 \\
\hline KF-CTRL & 0.64 & 0.63 & 0.60 & 0.50 & 0.28 \\
\hline PEF09 & 0.66 & 0.65 & 0.58 & 0.45 & 0.31 \\
\hline RATE01 & 0.64 & 0.65 & 0.62 & 0.52 & 0.30 \\
\hline RATE0001 & 0.71 & 0.61 & 0.42 & 0.30 & 0.24 \\
\hline EASYTRIG1 & 0.64 & 0.65 & 0.59 & 0.47 & 0.27 \\
\hline EASYTRIG2 & 0.64 & 0.63 & 0.58 & 0.46 & 0.28 \\
\hline $1 \mathrm{MIN}$ & 0.65 & 0.65 & 0.59 & 0.48 & 0.33 \\
\hline $15 \mathrm{MIN}$ & 0.66 & 0.65 & 0.60 & 0.51 & 0.32 \\
\hline CJAmod & 0.62 & 0.65 & 0.53 & 0.37 & 0.21 \\
\hline (d) POD & 0.1 & 1 & 2.5 & 5 & 10 \\
\hline KF-CTRL & 0.78 & 0.77 & 0.65 & 0.50 & 0.26 \\
\hline PEF09 & 0.80 & 0.77 & 0.64 & 0.45 & 0.29 \\
\hline RATE01 & 0.77 & 0.78 & 0.72 & 0.57 & 0.29 \\
\hline RATE0001 & 0.86 & 0.65 & 0.41 & 0.26 & 0.20 \\
\hline EASYTRIG1 & 0.84 & 0.80 & 0.67 & 0.49 & 0.25 \\
\hline EASYTRIG2 & 0.84 & 0.79 & 0.67 & 0.48 & 0.25 \\
\hline $1 \mathrm{MIN}$ & 0.83 & 0.78 & 0.66 & 0.48 & 0.29 \\
\hline $15 \mathrm{MIN}$ & 0.79 & 0.77 & 0.68 & 0.50 & 0.27 \\
\hline CJAmod & 0.88 & 0.76 & 0.54 & 0.33 & 0.18 \\
\hline (e) FAR & 0.1 & 1 & 2.5 & 5 & 10 \\
\hline KF-CTRL & 0.26 & 0.29 & 0.29 & 0.30 & 0.43 \\
\hline PEF09 & 0.24 & 0.26 & 0.31 & 0.36 & 0.42 \\
\hline RATE01 & 0.25 & 0.26 & 0.30 & 0.33 & 0.42 \\
\hline RATE0001 & 0.22 & 0.25 & 0.34 & 0.37 & 0.37 \\
\hline EASYTRIG1 & 0.29 & 0.29 & 0.31 & 0.37 & 0.40 \\
\hline EASYTRIG2 & 0.29 & 0.30 & 0.33 & 0.40 & 0.40 \\
\hline $1 \mathrm{MIN}$ & 0.28 & 0.28 & 0.30 & 0.30 & 0.29 \\
\hline $15 \mathrm{MIN}$ & 0.22 & 0.25 & 0.30 & 0.28 & 0.31 \\
\hline CJAmod & 0.33 & 0.27 & 0.26 & 0.29 & 0.44 \\
\hline (f) MAE & $0.1-2.5$ & $2.5-5$ & $5-10$ & $10-20$ & $>20$ \\
\hline KF-CTRL & 1.39 & 1.62 & 3.29 & 8.44 & 29.63 \\
\hline PEF09 & 1.53 & 1.49 & 3.40 & 8.39 & 29.88 \\
\hline RATE01 & 1.46 & 1.92 & 3.08 & 7.61 & 29.08 \\
\hline RATE0001 & 1.05 & 2.19 & 4.77 & 9.80 & 34.48 \\
\hline EASYTRIG1 & 1.39 & 1.57 & 3.14 & 8.25 & 30.42 \\
\hline EASYTRIG2 & 1.35 & 1.52 & 3.17 & 8.39 & 30.88 \\
\hline $1 \mathrm{MIN}$ & 1.45 & 1.51 & 3.36 & 8.56 & 30.51 \\
\hline $15 \mathrm{MIN}$ & 1.30 & 1.68 & 3.03 & 8.43 & 30.39 \\
\hline CJAmod & 1.23 & 2.00 & 4.51 & 9.54 & 32.66 \\
\hline (g) ME & $0.1-2.5$ & $2.5-5$ & $5-10$ & $10-20$ & $>20$ \\
\hline KF-CTRL & 0.80 & -1.26 & -2.82 & -7.12 & -29.59 \\
\hline PEF09 & 0.80 & -1.26 & -2.82 & -7.12 & -29.59 \\
\hline RATE01 & 0.82 & -0.63 & -2.07 & -7.22 & -29.06 \\
\hline RATE0001 & 0.52 & -1.34 & -4.42 & -9.35 & -34.38 \\
\hline EASYTRIG1 & 0.91 & -1.01 & -2.96 & -6.97 & -30.05 \\
\hline EASYTRIG2 & 0.87 & -0.95 & -2.95 & -6.88 & -30.66 \\
\hline $1 \mathrm{MIN}$ & 0.86 & -1.18 & -3.12 & -6.71 & -30.09 \\
\hline $15 \mathrm{MIN}$ & 0.72 & -1.18 & -2.48 & -6.66 & -30.39 \\
\hline CJAmod & 0.84 & -1.18 & -3.33 & -8.81 & -32.66 \\
\hline
\end{tabular}

www.nat-hazards-earth-syst-sci.net/11/1327/2011/ inspection of the Mean Absolute Error (MAE) (Table 1f) and Mean Error (ME) (Table 1g). All eight modifications and the control run underpredict the amounts of rain for all ranges (negative ME) with the exception of the first range of $0.1-$ $2.5 \mathrm{~mm}$. Between the modifications, RATE01 is characterized by the lowest values for the absolute error except for the range of $2.5-5.0 \mathrm{~mm}$.

Table 2 shows for every threshold and measure those modifications that have given better scores than the original KF scheme. In every cell of the table the modifications are sorted from better to worse but always better than the CTRL. Among the various modifications, it is obvious that for the rain/no-rain threshold, the modification RATE0001 gives the best scores for the measures CSI, HSS and FAR. The modification $15 \mathrm{MIN}$ predominates for the threshold of $1 \mathrm{~mm}$ while forcing the model to produce more precipitation and less cloud material by setting the parameter RATE equal to 0.1 , has a noticeably positive impact to the model's skill to produce medium and large amounts of precipitation (2.5 and $5 \mathrm{~mm})$. For very large amounts of rain $(>10 \mathrm{~mm})$, the modifications 1MIN and RATE01 give the best scores. More particularly, 1MIN outperforms the other modifications for the measures CSI, HSS, and FAR while RATE01 outperforms the other modifications for the measures B and POD. Overall, from Table 2 it is obvious that the RATE01 modification presents a consistently better skill than the other ones. Another remarkable finding in this table, with the exception of the 2.5 and $5 \mathrm{~mm}$, is the relatively low performance of the CTRL run.

Generally, for all selected cases the maximum precipitation was observed between 12:00 and 18:00 UTC (15:0021:00 LT) and during the next 6 -h period the precipitation was continuously decaying. Thus, forcing the model to produce more rainfall and less cloud material had a positive impact during the period of the verification procedure. On the other hand, in two cases (not shown here) where important precipitation amounts were observed close to midnight, the RATE0001 and CJAmod gave slightly better results in comparison with the RATE01. This is common for cases characterized by long-lived and organized convective systems where convective parameterization produces most of the rain without permitting more feedback cloud material for propagating convective cases. Hence these two modifications seem to be more useful for cases where rainfall is continued during the night. Anderson et al. (2007), using the alternative mass flux profile (CJAmod), found a similar improvement during nocturnal mesoscale precipitation events between the original and the modified KF CPS.

Finally, it would be interesting to investigate the impact of the aforementioned modifications on other model variables such as temperature. Trying to answer this question, the verification procedure was repeated for the temperature at $2 \mathrm{~m}$. This analysis showed that the verification scores of temperature from the CTRL experiments do not differ from those of the experiments with modified KF scheme. Namely the 
Table 2. List of modifications (for each threshold and statistical measure) that give better scores that the original KF scheme. The list of modifications is sorted from the best to the worst. The modification RATE01 is in bold in order to emphasize the fact that it has given good results for the majority of the thresholds and measures.

\begin{tabular}{|c|c|c|c|c|c|}
\hline & 0.1 & 1 & 2.5 & 5 & 10 \\
\hline B & $\begin{array}{l}\text { 15MIN } \\
\text { RATE01 } \\
\text { PEF09 }\end{array}$ & $\begin{array}{l}\text { 15MIN } \\
\text { RATE0001 } \\
\text { RATE01 } \\
\text { CJAmod } \\
\text { PEF09 }\end{array}$ & $\begin{array}{l}\text { 1MIN } \\
\text { PEF09 }\end{array}$ & $\begin{array}{l}\text { RATE01 } \\
\text { EASYTRIG2 } \\
\text { EASYTRIG1 }\end{array}$ & $\begin{array}{l}\text { RATE01 } \\
\text { EASYTRIG1 } \\
\text { PEF09 } \\
\text { EASYTRIG2 }\end{array}$ \\
\hline CSI & $\begin{array}{l}\text { RATE0001 } \\
\text { 15MIN } \\
\text { 1MIN } \\
\text { EASYTRIG1 } \\
\text { PEF09 } \\
\text { EASYTRIG2 }\end{array}$ & $\begin{array}{l}\text { 15MIN } \\
\text { PEF09 } \\
\text { RATE01 } \\
\text { 1MIN } \\
\text { CJAmod } \\
\text { EASYTRIG1 }\end{array}$ & & RATE01 & $\begin{array}{l}\text { 1MIN } \\
15 \mathrm{MIN} \\
\text { PEF09 } \\
\text { RATE01 }\end{array}$ \\
\hline HSS & $\begin{array}{l}\text { RATE0001 } \\
\text { PEF09 } \\
15 \mathrm{MIN} \\
1 \mathrm{MIN}\end{array}$ & $\begin{array}{l}\text { 15MIN } \\
\text { CJAmod } \\
\text { PEF09 } \\
\text { RATE01 } \\
\text { EASYTRIG1 } \\
\text { 1MIN }\end{array}$ & RATE01 & $\begin{array}{l}\text { RATE01 } \\
\text { 15MIN }\end{array}$ & $\begin{array}{l}1 \mathrm{MIN} \\
15 \mathrm{MIN} \\
\text { PEF09 } \\
\text { RATE01 }\end{array}$ \\
\hline POD & $\begin{array}{l}\text { CJAmod } \\
\text { RATE0001 } \\
\text { EASYTRIG2 } \\
\text { EASYTRIG1 } \\
\text { 1MIN } \\
\text { PEF09 } \\
\text { 15MIN }\end{array}$ & $\begin{array}{l}\text { EASYTRIG1 } \\
\text { EASYTRIG2 } \\
\text { 1MIN } \\
\text { RATE01 }\end{array}$ & $\begin{array}{l}\text { RATE01 } \\
\text { 15MIN } \\
\text { EASYTRIG1 } \\
\text { EASYTRIG2 } \\
\text { 1MIN }\end{array}$ & RATE01 & $\begin{array}{l}\text { RATE01 } \\
\text { PEF09 } \\
1 \mathrm{MIN} \\
15 \mathrm{MIN}\end{array}$ \\
\hline FAR & $\begin{array}{l}\text { RATE0001 } \\
\text { 15MIN } \\
\text { PEF09 } \\
\text { RATE01 }\end{array}$ & $\begin{array}{l}\text { RATE0001 } \\
\text { 15MIN } \\
\text { RATE01 } \\
\text { PEF09 } \\
\text { CJAmod } \\
\text { 1MIN }\end{array}$ & CJAmod & $\begin{array}{l}\text { 15MIN } \\
\text { CJAmod }\end{array}$ & $\begin{array}{l}\text { 1MIN } \\
\text { 15MIN } \\
\text { RATE0001 } \\
\text { EASYTRIG1 } \\
\text { EASYTRIG2 } \\
\text { RATE01 } \\
\text { PEF09 }\end{array}$ \\
\hline
\end{tabular}

MAE (averaged over all stations at 12:00 and 18:00 UTC and for all cases) ranged from 1 to 1.03 , while the ME ranged from -0.52 to -0.27 .

\section{Example of case study}

A more detailed analysis of the event of 30 August 2009 is presented in the following. Although, one of the twenty aforementioned warm events could have been selected, this one was selected since during 2009, the raingauge network density around Greece was developed remarkably, especially in mountainous regions. Hence, 132 raingauges were available for the statistical verification of the specific event. 30 August 2009 was a typical summer day characterized by widespread convective activity above continental Greece during noon. Figure 2 depicts the lightning activity during the period 12:00-18:00 UTC as it was recorded from the ZEUS network (Kotroni and Lagouvardos, 2008; Lagouvardos et al., 2009), showing intense lightning activity over the central part of Greece, Epirus, Northern Ionian Sea, Central Peloponnissos and part of Macedonia (locations are shown in Fig. 1b). Figure 3 depicts the cloud top temperatures at 13:30 UTC along with the lighting activity detected during the $15 \mathrm{~min}$ preceding that time. The stations where the 6$\mathrm{h}$ accumulated precipitation exceeded $8 \mathrm{~mm}$ during the period 12:00-18:00 UTC, are marked by the yellow stars in the same figure. Rain gauge measurements showed that the major part of the rain was observed between 13:00 and 14:00 UTC (16:00-17:00 LT) near the time of maximum surface heating.

Following the same methodology used in Sect. 5, the statistical scores for the same thresholds have been calculated and are presented in Table 3. This table shows for every 
Table 3. This table shows for every threshold and measure which of the selected modifications gives the best or equal score than the original KF scheme.

\begin{tabular}{|c|c|c|c|c|c|}
\hline & 0.1 & 1 & 2.5 & 5 & 10 \\
\hline B & RATE0001 = 1.05 & PR & EASYTRIG2 =0.92 & RATE01 $=0.89$ & KF-CTRL RATE01 $=0.64$ \\
\hline CSI & RATE0001 $=0.86$ & $\begin{array}{l}\text { EASYTRIG2, } \\
15 \mathrm{MIN}=0.83\end{array}$ & EASYTRIG1 = 0.71 & $\begin{array}{l}\text { CTRL }=\text { PEF09 } \\
=\text { EASYTRIG } 1 \\
=1 \mathrm{MIN}=15 \mathrm{MIN} \\
=0.63\end{array}$ & $\mathrm{PEF09}=0.45$ \\
\hline HSS & RATE0001 $=0.89$ & $\begin{array}{l}\text { EASYTRIG2, } \\
15 \mathrm{MIN}=0.83\end{array}$ & EASYTRIG1 $=0.80$ & $\begin{array}{l}\text { CTRL = PEF09 } \\
=\text { EASYTRIG } 1 \\
=1 \mathrm{MIN}=15 \mathrm{MIN} \\
=0.74\end{array}$ & $\mathrm{PEF09}=0.60$ \\
\hline POD & CJA2nd $=1$ & $\begin{array}{l}\text { EASYTRIG2, } \\
15 \mathrm{MIN}=0.82\end{array}$ & $\begin{array}{l}\text { EASYTRIG1, } \\
\text { EASYTRIG2 = } 0.77\end{array}$ & $\begin{array}{l}\text { CTRL }=\text { PEF09 }=\text { RATE01 } \\
=\text { EASYTRIG } 1=\text { EASYTR } 2 \\
=1 \mathrm{MIN}=15 \mathrm{MIN} \\
=\text { RATE01-PEF09 }=0.67\end{array}$ & KF-CTRL PEF09 = 0.45 \\
\hline FAR & CTRL 15MIN & $\begin{array}{l}\text { CTRL }=\text { PEF09 } \\
=\text { EASYTRIG1 } \\
=\text { EASYTRIG2 } \\
=15 \mathrm{MIN}=0.07\end{array}$ & RATE0001 $=0$ & RATE0001 = CJA2nd = 0 & $\begin{array}{l}\text { PEF09 = 15MIN, } \\
1 \mathrm{MIN}, \\
\text { CJA2nd =0 }\end{array}$ \\
\hline
\end{tabular}

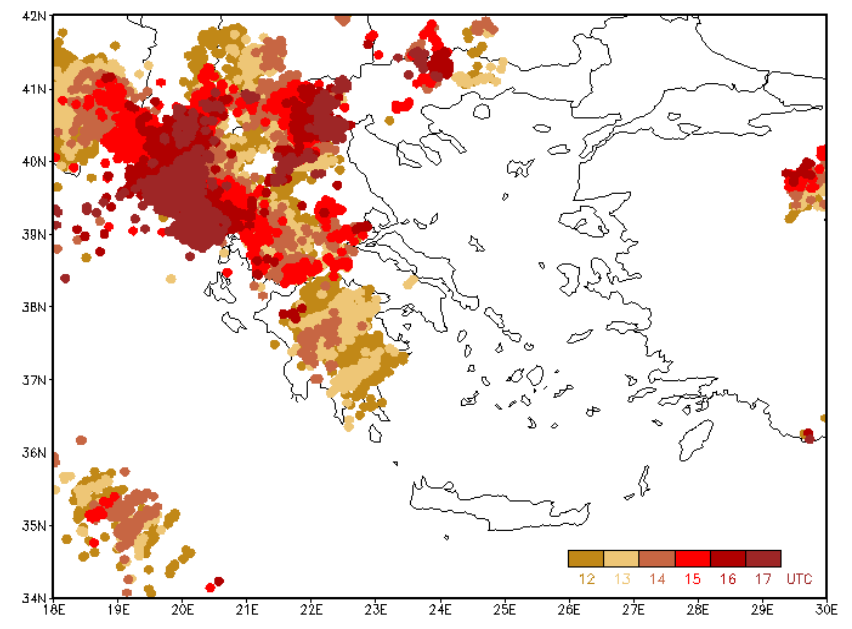

Fig. 2. Lighting activity over Greece during the period 12:0018:00 UTC 30 August 2009 as detected by the ZEUS network.

threshold and measurement which of the selected modifications gives better or equal scores than the original $\mathrm{KF}$ scheme. Between the various modifications, one more has been added, which is the combination of the RATE01 and PEF09 (RP), which showed the best performance according to the analysis of Sect. 5. The main characteristic of these two modifications is the fact that they force the scheme to produce more precipitation than the original scheme and as a result, this combination may alleviate the model's tendency to underpredict precipitation during the warm season's convective events. Hence, as far as Frequency Bias is concerned there is great variability between the various thresholds:

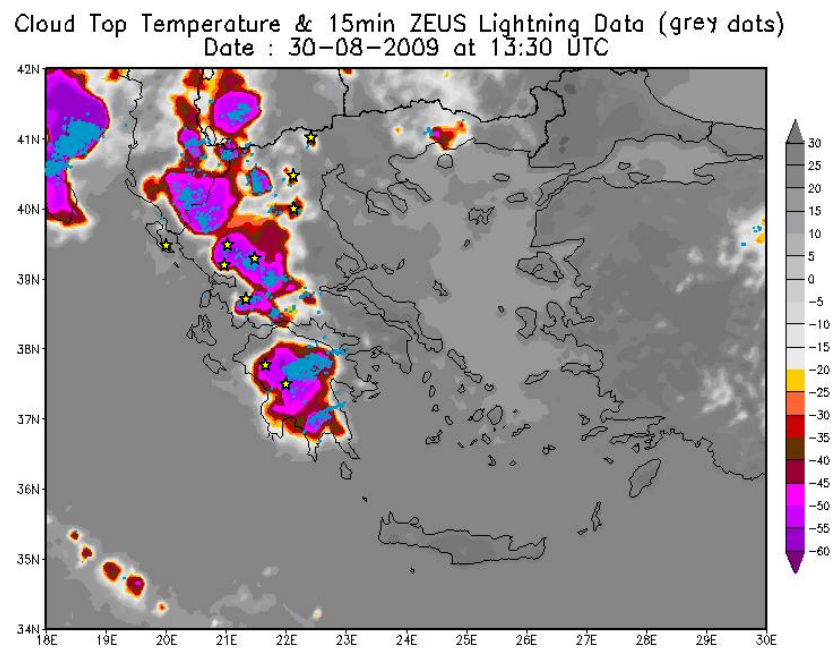

Fig. 3. The cloud top temperature at 13:30 UTC, 30 August 2009, overlaid with the lightning activity (blue dots) during the preceeding $15 \mathrm{~min}$. The stations where the $6-\mathrm{h}$ accumulated precipitation exceeded $8 \mathrm{~mm}$ during the period 12:00-18:00 UTC are marked by the yellow stars.

as for the rain/no rain threshold, the RATE0001 gives the best score (1.05) while for the medium precipitation (1 and $2.5 \mathrm{~mm}$ ), the PR gives the best results ( 1 and 0.92 , respectively). For the heavy precipitation, there is a clear predominance of the RATE01 ( 0.89 for the $5 \mathrm{~mm}$ and 0.64 for the $10 \mathrm{~mm}$, respectively), while the original scheme has the same score for the threshold of $10 \mathrm{~mm}$. As far as the CSI, for the rain/no-rain threhold the modification RATE0001 continues to have the best performance while EASYTRIG2 and 15MIN 

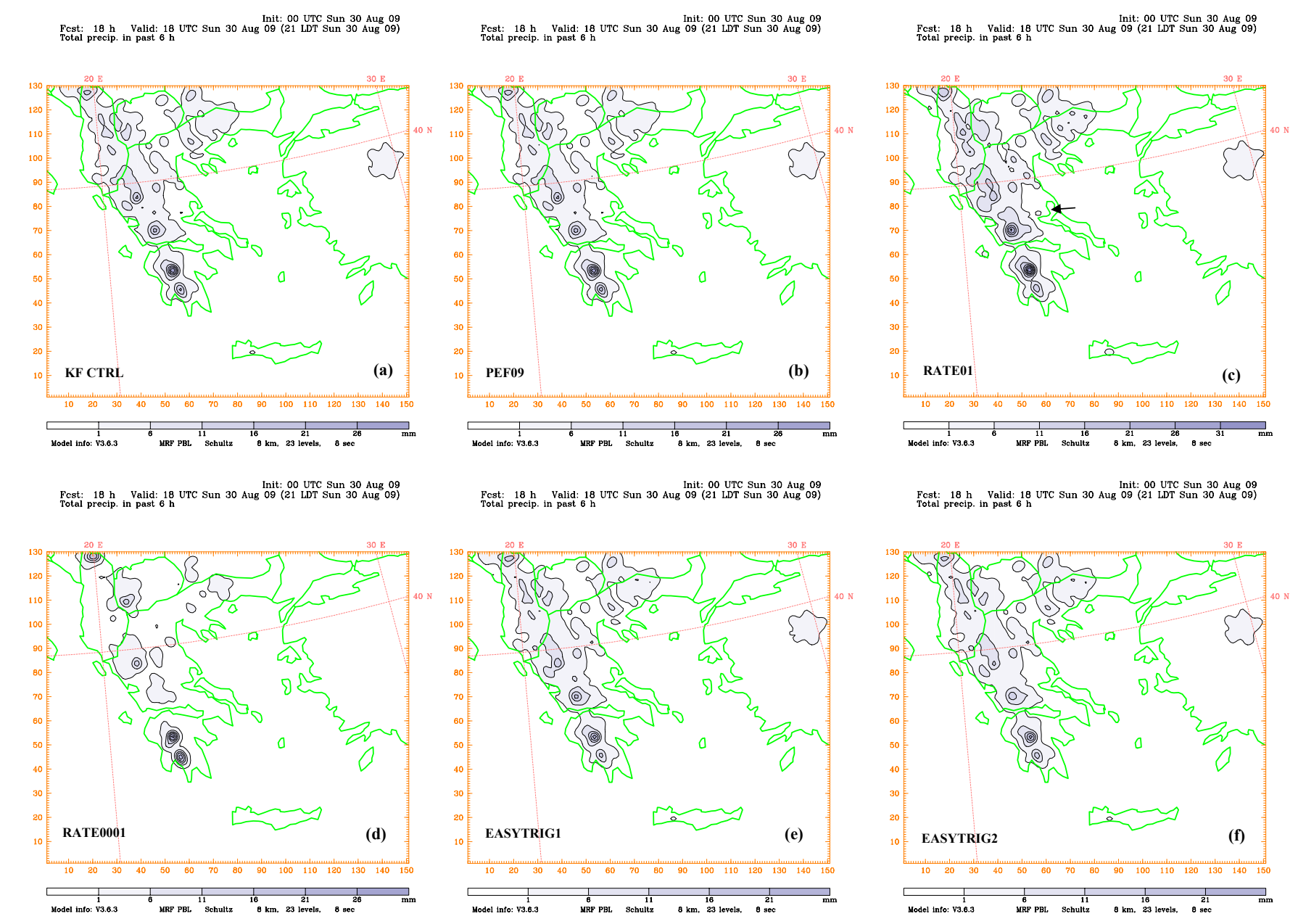

Fig. 4. 6-h accumulated precipitation from $t+12$ up to $t+18$ during the period 12:00-18:00 UTC 30 August 2009 from the MM5 simulation using the (a) unmodified version of KF scheme, (b) PEF09, (c) RATE01, (d) RATE0001, (e) EASYTRIG1, (f) EASYTRIG2, (g) 1MIN, (h) $15 \mathrm{MIN}$, (i) CJAmod and (j) PR modification.

give the best score for the $1 \mathrm{~mm}(0.83)$ and EASYTRIG1 gives the best score for the $2.5 \mathrm{~mm}(0.71)$. For the $5 \mathrm{~mm}$ the differences between the modifications are indiscernible while PEF09 gives the best score (0.45) for the large amounts of precipitation. The results are similar for HSS also. In addition, the implementation of CJAmod presents the best POD for the rain/no-rain threshold, but this value is combined with very large values for the B (1.42, not shown in this table). On the other hand, the predominance of the EASYTRIG1 and EASYTRIG2 modifications remains for the next two thresholds. For heavy precipitation $(>10 \mathrm{~mm})$, the original and the PEF09 performs better $(0.45)$ than the other modifications. The FAR is equal to zero for the threshold of $2.5 \mathrm{~mm}$ when RATE0001 is implemented, for the threshold of $5 \mathrm{~mm}$ when RATE0001 and CJAmod are implemented. For the large amount of rain four modifications gives a zero false alarm ratio.

The panel in Fig. 4 shows the 6-h accumulated precipitation from $t+12$ up to $t+18$ during the period 12:00-
18:00 UTC. In general irrespective of the choice of modification, the forecasted precipitation is in close agreement with the observed lightning activity presented in Fig. 2. However the model fails to simulate the high amount of rainfall $(33.2 \mathrm{~mm})$ that occurred on the Island of Corfu in the North Ionian Sea (see Fig. 1b). On the other hand, it is obvious that in comparison with CTRL, the modifications RATE01, 15MIN, CJAmod and PR increase the maximum values of precipitation by $5-10 \mathrm{~mm}$. Moreover, the modifications RATE01, 15MIN and PR succeed in simulating the observed precipitation in the eastern continental Greece (see the black arrows in the Fig. $4 c$, h, and j) where significant lightning activity was detected.

As it was also mentioned in Sect. 5, the RATE01 had the best performance for the mean error (ME). Table 4 shows the observed accumulated precipitation between 12:0018:00 UTC (2nd column), the forecasted precipitation for the same period using the original KF scheme (3rd column), the forecasted precipitation using the modification RATE01 (4th 

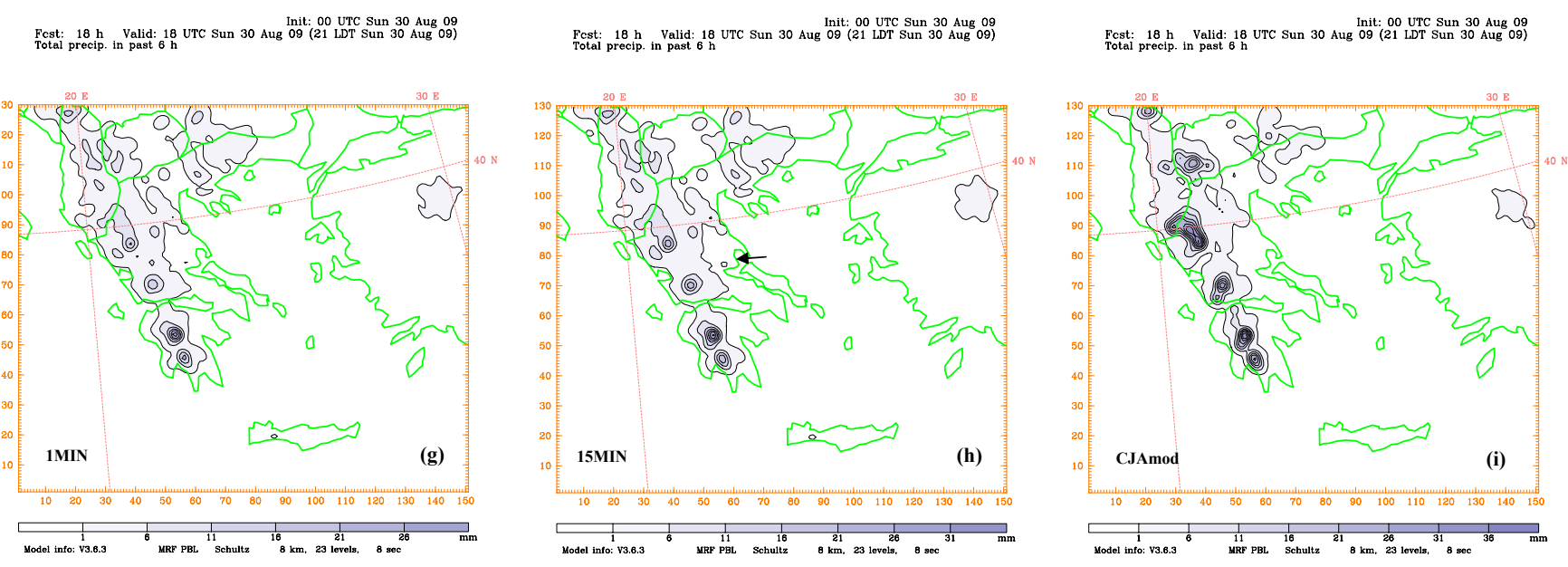

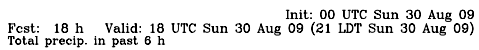

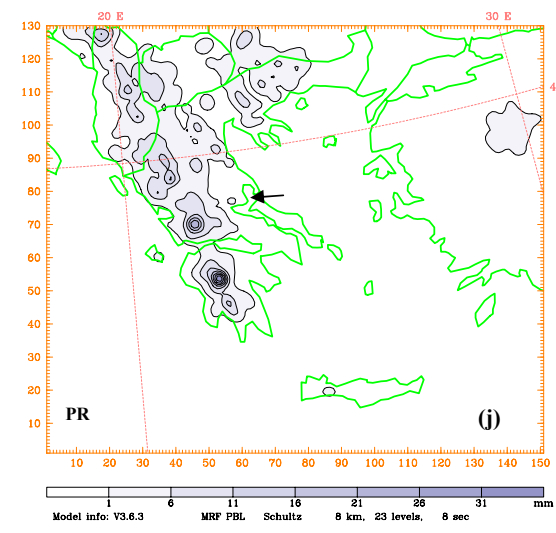

Fig. 4. Continued.

column) and PR (5th column) for 11 selected stations. With the exception of Corfu's station where the model was unable to simulate the observed precipitation, in the other stations the use of the modifications seems to have a positive effect on the values of quantitative precipitation. For example, the observed accumulated precipitation in the station of Foloi was $22.2 \mathrm{~mm}$. The use of the original scheme gives only $10.4 \mathrm{~mm}$ while the modification RATE01 and PR give 18.6 and 19.0, respectively. With the exception of the Agrinio and Arta stations, where the original scheme gives slightly better results, there is a clear improvement using the two modifications.

\section{Summary and discussion}

In the frame of this work, the sensitivity of MM5 model forecasts of warm season precipitation to the various modifications of the KF CPS for 20 selected cases over Greece is examined. The modifications include: (i) the maximization of the convective scheme precipitation efficiency (PEF09). In this modification, the variability of PE has been removed and it is consistently equal to 0.9 , which is the maximum value. (ii) The change of the convective time step to 1 (1MIN) and to 15 (15MIN) minutes respectively. In this modification, the period at which the model calls the convective parameterization scheme has been changed from 5 min to 1 and $15 \mathrm{~min}$, respectively. (iii) The forcing of the convective scheme to produce more/less cloud material (RATE01 and RATE0001). In this modification the model sensitivity to the production of more or less rainfall and cloud material is checked. Hence, two different values $(-0.1$ and -0.001$)$ are used in the equation which is used to the calculation of precipitation. (iv) Changes to the trigger function (EASYTRIG1 and EASYTRIG2). In this modification, two different coefficients ( 0.5 and 1.0) have been added to the equation that describe the main criterion which the scheme activation is based upon, and (v) the alteration of the vertical profile of updraft mass flux detrainment (CJAmod). In this modification, the updraft mass flux is reduced linearly from Level of Free Sink instead of the Equilibrium Level, to the Cloud Top. 
Table 4. Observed accumulated precipitation between 12:00 and 18:00 UTC, 30 August 2009 (2nd column), forecasted precipitation for the same period using the original KF scheme (3rd column), forecasted precipitation using the modification RATE01 (4th column) and the PR (5th column) for 11 selected stations.

\begin{tabular}{lcrrr}
\hline Station & $\begin{array}{c}\text { Precipitation } \\
\text { between 12:00 } \\
\text { and 18:00 UTC }\end{array}$ & KF-CTRL & RATE01 & $\begin{array}{c}\text { RATE01 } \\
\text { PEF09 }\end{array}$ \\
\hline Agrinio & 14.0 & 11.1 & 9.9 & 0.4 \\
Arta & 9.2 & 8.4 & 8.3 & 7.8 \\
Derviziana Ioan. & 12.0 & 10.5 & 11.7 & 11.9 \\
Foloi & 22.2 & 10.4 & 18.6 & 19.0 \\
Corfu & 33.2 & 0.0 & 0.0 & 0.0 \\
Koniskos & 16.2 & 15.1 & 15.2 & 15.4 \\
Megalopoli & 9.8 & 2.8 & 4.6 & 4.6 \\
Vegoritida & 13.8 & 6.3 & 6.7 & 6.9 \\
Veroia & 13.2 & 1.8 & 2.4 & 2.1 \\
Miliana Arta & 19.5 & 5.6 & 8.9 & 9.2 \\
Pistiana Arta & 34.9 & 12.9 & 16.6 & 18.8 \\
\hline
\end{tabular}

One hundred eighty simulations were carried out on two nested domains, with horizontal grid increments of 24 and $8 \mathrm{~km}$, respectively. The verification dataset consists of ground precipitation measurements from 113 rain gauges. For verification purposes, the measured and predicted precipitation amounts within a given 6-h forecast period $(t+12$ up to $t+18$ ) were compared for the following precipitation thresholds: 0.1, 1.0, 2.5, 5.0 and $10.0 \mathrm{~mm}$.

In general, during the warm season the forecasts suffer from underestimation of the volumetric precipitation in the areas where moderate and high precipitation occurs. This problem can be alleviated when the model is forced to produce more precipitation and/or less cloud material. With the exception of RATE0001 and CJAmod, all the other modifications force the model to produce more rain than the original scheme. At this point it should be noted that forcing the model to produce more rainfall is supported by the importance of improving the precipitation forecasts of the high amounts, as those are producing the most important societal impacts. For the 20 selected cases, the modification RATE01 gives the best statistical scores for the moderate and high precipitation amounts, while the same modification and the $1 \mathrm{MIN}$ improves the model performance for very high precipitation $(>10 \mathrm{~mm})$. In order to provide a representative way about the performance of each modification, Fig. 5 has been plotted. This figure summarises the number of times that each modification gives better results than the original KF scheme, irrespectively of the threshold and statistical measure. It is obvious that the RATE01 modification gives the best results for the summertime convective precipitation forecasts, and thus this modification could be introduced into the operational chain for this period of the year.

In the present study the verification procedure focuses on the quantitative precipitation forecasts. Nevertheless, it was

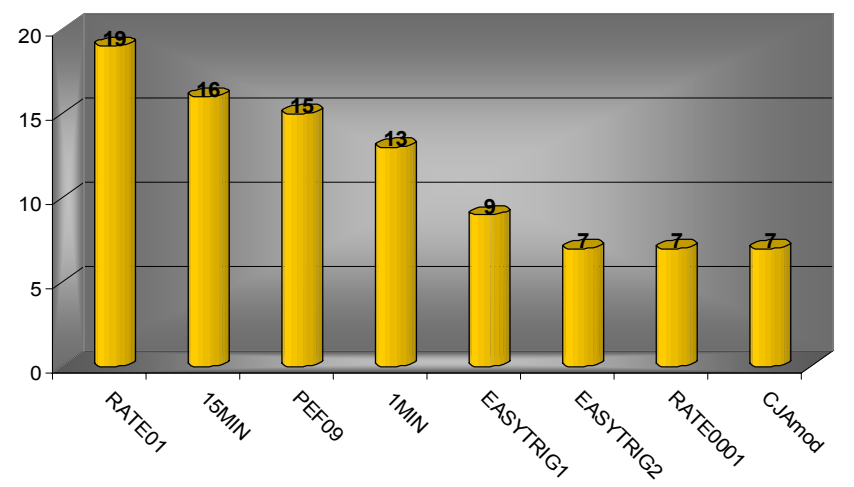

Fig. 5. Synopsis of all case studies: numbers on top of each column show how many times each modification gives better results than the original KF scheme, irrespectively of the threshold and statistical measure.

found that the investigated modifications do not have any statistically significant impact on other parameters, such as temperature.

Finally, it would be very interesting to study also the interaction of the convective parameterization scheme with the explicit microphysics. This would be possible if this study were expanded for cold period cases for which stratiform and convective precipitation are both important.

Acknowledgements. This work has been supported by the EU financed project FLASH (Contract No. 516172). The Hellenic National Meteorological Service, the Hellenic Ministry of Rural and Development and Food, the National Technical University of Athens and the University of Ioannina are kindly acknowledged for providing the observed precipitation data used in this study. The authors are also grateful to NCEP (USA) for providing GFS initial and forecast field data, which allowed the operational meteorological forecasting at the National Observatory of Athens.

Edited by: R. Deidda

Reviewed by: two anonymous referees

\section{References}

Akylas, E., Kotroni, V., and Lagouvardos, K.: Sensitivity of high resolution operational weather forecasts to the choice of the planetary boundary layer scheme, Atmos. Res., 84(1), 49-57, doi:10.1016/j.atmosres.2006.06.001, 2007.

Anderson, C. J., Gallus Jr., W. A., Arritt, R. W., and Kain, J. S.: Impact of adjustments in the Kain-Fritsch convective scheme on QPF of elevated convection, in: 19th Conf. on Weather Analysis and Forecasting/15th Conf. on Numerical Weather Prediction, Amer. Meteor. Soc., 12-16 August 2002, San Antonio, Texas, 2002.

Anderson, C. J., Arritt, R. W., and Kain, J. S.: An alternative mass flux profile in the Kain-Fritsch convective parameterization and its effects in seasonal precipitation, J. Hydrometeorol., 8, 1128$1140,2007$. 
Bechtold, P., Bazile, E., Guichard, F., Mascart, P., and Richard, E.: A mass-flux convection scheme for regional and global models, Q. J. Roy. Meteor. Soc., 127(573), 869-886, doi:10.1002/qj.49712757309, 2001.

Correia, J., Arritt, R. W., and Anderson, C. J.: Idealized mesoscale convective system structure and propagation using convective parameterization, Mon. Weather Rev., 136, 2422-2442, doi:10.1175/2007MWR2229.1, 2008 .

Davis, C. A., Manning, K. W., Carbone, R. E., Trier, S. B., and Tuttle, J. D.: Coherence of warm-season continental rainfall in numerical weather prediction models, Mon. Weather Rev., 131, 2667-2679, 2003.

Dudhia, J.: A nonhydrostatic version of the Penn State-NCAR Mesoscale Model - Validation tests and simulation of an Atlantic cyclone and cold front, Mon. Weather Rev., 121, 1493-1513, 1993.

Emanuel, K. A.: A scheme for representing cumulus convection in large-scale models, J. Atmos. Sci., 48, 2313-2335, 1991.

Emanuel, K. A. and Zivkovic-Rothman, M.: Development and evaluation of a convection scheme for use in climate models, J. Atmos. Sci., 56, 1766-1782, 1999.

Ferrier, B. S.: Modifications of Two Convective Schemes used in the NCEP Eta Model, 16th Conference on Numerical Weather Prediction, Seattle, WA, 11-15 January 2004.

Flocas, H. A.: Contribution Study on the Problem of Cyclogenesis over the Aegean Sea, Ph.D. thesis, University of Thessaloniki, Greece, 200 pp., 1993.

Fritsch, J. M. and Chappell, C. F.: Numerical prediction of convectively driven mesoscale pressure systems. Part I: Convective parameterization, J. Atmos. Sci., 37, 1722-1732, 1980.

Gallus, W. A. and Segal, M.: Impact of improved initialization of mesoscale features on convective system rainfall in 10-km Eta simulations, Weather Forecast., 16, 680-696, 2001.

Grell, G. A.: Prognostic evaluation of assumptions used by cumulus parameterizations, Mon. Weather Rev., 121, 764-787, 1993.

Grell, G. A., Dudhia, J., and Stauffer, D. R.: A description of the fifth generation Penn State/NCAR Mesoscale Model (MM5), NCAR Tech. Note NCAR/TN-3801STR, 138 pp., 1994.

Hong, S.-Y. and Pan, H.-L.: Nonlocal boundary layer vertical diffusion in a medium-range forecast model, Mon. Weather Rev., 124(10), 2322-2339 1996.

Kain, J. S.: The Kain-Fritch convective parameterization: an update, J. Appl. Meteorol., 43, 170-181, 2004.

Kain, J. S. and Fritsch, M.: A one-dimensional entraining/detraining plume model and its application in convective parameterization, J. Atmos. Sci., 47(23), 2784-2802, 1990.

Kain, J. S. and Fritsch, M.: The role of the convective "triggering function" in numerical forecasts of mesoscale convective systems, Meteorol. Atmos. Phys., 49(1-4), 93-106, doi:10.1007/BF01025402, 1992.

Kain, J. S. and Fritsch, M.: Convective parameterization for mesoscale models: The Kain-Fritsch scheme. The representation of cumulus convection in numerical models, Amer. Meteor. Soc., Meteor. Monogr., 24, 165-170, 1993.

Kostopoulou, E. and Jones, P. D.: Comprehensive analysis of the climate variability in the eastern Mediterranean. Part I: Map pattern classification. International, J. Climatol., 27(9), 1189-1214, doi:10.1002/joc.1467, 2007.
Kotroni, V. and Lagouvardos, K.: Precipitation forecast skill of different convective parameterization and microphysical schemes: Application for the cold season over Greece, Geophys. Res. Lett., 28(10), 1977-1980, doi:10.1029/2000GL012705, 2001.

Kotroni, V. and Lagouvardos, K.: Evaluation of MM5 high-resolution real-time forecasts over the urban area of Athens, Greece, J. Appl. Meteorol., 43, 1666-1678, doi:10.1175/JAM2170.1, 2004.

Kotroni, V. and Lagouvardos, K.: Lightning occurrence in relation with elevation, terrain slope and vegetation cover over the Mediterranean, J. Geophys. Res.-Atmos., 113, D21118, doi:10.1029/2008JD010605, 2008.

Kuo, Y.-H., Reed, R. J., and Liu, Y.: The ERICA IOP5 storm. Part III: Mesoscale cyclogenesis and precipitation parameterization, Mon. Weather Rev., 124(7), 1409-1434 1996.

Lagouvardos, K., Kotroni, V., Betz, H.-D., and Schmidt, K.: A comparison of lightning data provided by ZEUS and LINET networks over Western Europe, Nat. Hazards Earth Syst. Sci., 9, 1713-1717, doi:10.5194/nhess-9-1713-2009, 2009.

Mazarakis, N., Kotroni, V., Lagouvardos, K., and Argiriou, A.: Storms and lightning activity in Greece during the warm period of 2003-06, J. Appl. Meteorol. Clim., 47(12), 3089-3098, doi:10.1175/2008JAMC1798.1, 2008.

Mazarakis, N., Kotroni, V., Lagouvardos, K., and Argiriou, A.: The sensitivity of numerical forecasts to convective parameterization during the warm period and the use of lightning data as an indicator for convective occurrence, Atmos. Res., 94(4), 704-714, doi:10.1016/j.atmosres.2009.03.002, 2009.

Ogura, Y. and Cho, H. R.: Diagnostic determination of cumulus cloud populations from observed large-scale variables, J. Atmos. Sci., 30(7), 1276-1286 1973.

Ridout, J. A., Jin, Y., and Liou, C. S.: A cloud-base quasibalance constraint for parameterized convection: Application to the Kain-Fritsch cumulus scheme, Mon. Weather Rev., 133, 3315-3334, 2005.

Spencer, P. L. and Stensrud, D. J.: Simulating flash flood events: Importance of the subgrid representation of convection, Mon. Weather Rev., 126(11), 2884-2912, 1998.

Schultz, P.: An explicit cloud physics parameterization for operational numerical weather prediction, Mon. Weather Rev., 123, 3331-3343, 1995.

Trigo, I. F., Bigg, G. R., and Davies, T. D.: A climatology of cyclogenesis mechanisms in the Mediterranean, Mon. Weather Rev., 130, 549-569, 2002.

Truong, N. M., Tien, T. T., Pielke Sr., R. A., Castro, C. L., and Leoncini, G.: A modified Kain-Fritsch scheme and its application for simulation of an extreme precipitation event in Vietnam, Mon. Weather Rev., 137, 766-789, 2009.

Wang, W. and Seaman, N. L.: A Comparison Study of Convective Parameterization Schemes in a Mesoscale Model, Mon. Weather Rev., 125, 252-278, 1997.

WMO: Recommendations for the verification and intercomparison of QPFs and PQPFs from Operational NWP Models, WMO/TD - No. 1485, 2008.

Yang, Z. and Arritt, R. W.: Ensemble simulations of regional flood and drought climates. Preprints, Precipitation Extremes: Prediction, Impacts, and Responses, Albuquerque, NM, Amer. Meteor. Soc., 236-239 2001. 